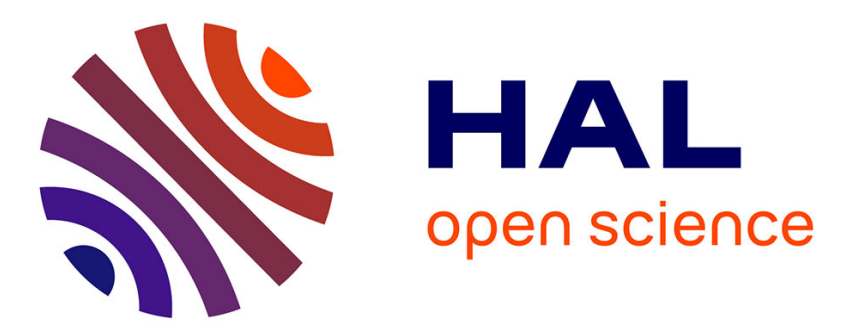

\title{
Montage de compression à vitesse constante pour très hautes températures
}

\author{
J.L. Cadoz, J. Castaing, C. Dolin, H. Gervais, B. Pellissier
}

\section{To cite this version:}

J.L. Cadoz, J. Castaing, C. Dolin, H. Gervais, B. Pellissier. Montage de compression à vitesse constante pour très hautes températures. Revue de Physique Appliquée, 1975, 10 (4), pp.247-249. 10.1051/rphysap:01975001004024700 . jpa-00243910

\section{HAL Id: jpa-00243910 https://hal.science/jpa-00243910}

Submitted on 1 Jan 1975

HAL is a multi-disciplinary open access archive for the deposit and dissemination of scientific research documents, whether they are published or not. The documents may come from teaching and research institutions in France or abroad, or from public or private research centers.
L'archive ouverte pluridisciplinaire HAL, est destinée au dépôt et à la diffusion de documents scientifiques de niveau recherche, publiés ou non, émanant des établissements d'enseignement et de recherche français ou étrangers, des laboratoires publics ou privés. 


\begin{abstract}
Classification
Physics Abstracts

0.622

\section{MONTAGE DE COMPRESSION A VITESSE CONSTANTE POUR TRẼS HAUTES TEMPÉRATURES}

\author{
J. L. CADOZ, J. CASTAING, C. DOLIN, H. GERVAIS et B. PELlissier
}

Labotatoire de Physique des Matériaux, C. N. R. S. Bellevue, 92190 Meudon, France

(Reçu le 27 janvier 1975, révisé le 17 février 1975, accepté le 28 février 1975)

\begin{abstract}
Résumé. - Un appareillage pour essais de déformation à haute température (jusqu'à $1800^{\circ} \mathrm{C}$ ) sous atmosphère contrôlée, en compression à vitesse de traverse constante a été construit sur une machine INSTRON. Pour connaître avec précision la déformation de l'échantillon, un système d'extensométrie a été placé. Deux courbes contrainte-déformation faites sur de l'alumine polycristalline à deux températures différentes sont données.

Abstract. - We describe an apparatus for high temperature (up to $1800{ }^{\circ} \mathrm{C}$ ) constant-speed crosshead compression in a controlled atmosphere, built in an Instron machine. An extensometer device is used in order to determine accurate values of the strain. Two stress-strain curves for polycristalline alumina are shown for two different temperatures.
\end{abstract}

1. La déformation en compression est une méthode intéressante pour étudier la plasticité de matériaux céramiques. En effet, ceux-ci sont généralement fragiles et le taux de déformation que l'on atteint en compression est supérieur à celui obtenu par traction (propagation de fissures plus facile dans ce dernier cas).

Ce mode de sollicitation ne nécessite aucun système de fixation de l'éprouvette et celle-ci peut être de petite taille, ce qui facilite sa préparation, évite d'utiliser des grandes quantités de matériau, et permet d'avoir une température constante le long de l'éprouvette.

Le montage de type direct [1] nous a semblé être le plus rigide et le plus simple à réaliser.

2. Description. - 2.1 Machine De DéFormation. - Le montage est fait dans une machine INSTRON (Fig. 1).

La déformation de l'échantillon de dimension moyenne $(3 \times 3 \times 6 \mathrm{~mm})$ se fait par déplacement à vitesse constante de la poutre mobile à laquelle est fixé le piston de compression supérieur $\left({ }^{1}\right)$. Le piston inférieur est fixé sur la cellule de compression. Ces deux pistons sont à l'intérieur d'un moufle permettant de travailler sous atmosphère contrôlée - en particulier sous pression d'oxygène. La liaison entre le moufle solidaire de la partie supérieure mobile et la partie inférieure fixe se fait par un soufflet (Fig. 1).

(1) Les pistons sont en alumine AF 975 DESMARQUEST.
Le four en chromite de lanthane [2] permet de travailler jusqu'à $1800^{\circ} \mathrm{C}$, la régulation de température annoncée par le constructeur se fait à $\pm 0,1^{\circ}$. Nous avons vérifié ce point en utilisant le défaut du montage qui est sa grande sensibilité à la dilatation thermique des pistons : en chargeant légèrement le montage, on ne constate pas de dérive de charge, la limite de résolution étant de $1 \mathrm{~N}$, cela correspond à une dérive de température inférieure à $1 / 10^{\circ}$. Le profil thermique obtenu avec le montage de déformation est donné figure 2 . La résistance mécanique des pistons d'alumine polycristalline s'effondre aux environs de $1600^{\circ} \mathrm{C}$ (Fig. 3). Aussi pour des températures supérieures à $1600^{\circ} \mathrm{C}$, il est nécessaire de prolonger par des blocs monocristallins de saphir $\left(^{2}\right)$ (axe c parallèle à l'axe de compression) les pistons en alumine polycristalline (Fig. 2).

Ces deux blocs monocristallins étant des pièces onéreuses, nous avons placé entre eux et l'échantillon, de part et d'autre de celui-ci, une plaquette monocristalline de saphir de même orientation. Au cours de nos expériences sur $\mathrm{Al}_{2} \mathrm{O}_{3}-\alpha$ [3] l'indentation est évitée si la désorientation de ces plaquettes avec l'axe $\mathbf{c}$ est inférieure à $1^{\circ}$ et si leur épaisseur est supérieure à $4 \mathrm{~mm}$.

(2) Les monocristaux d'alumine (non usinés) sont fournis par le Rubis synthétique des Alpes 38 Jarrie (France). 


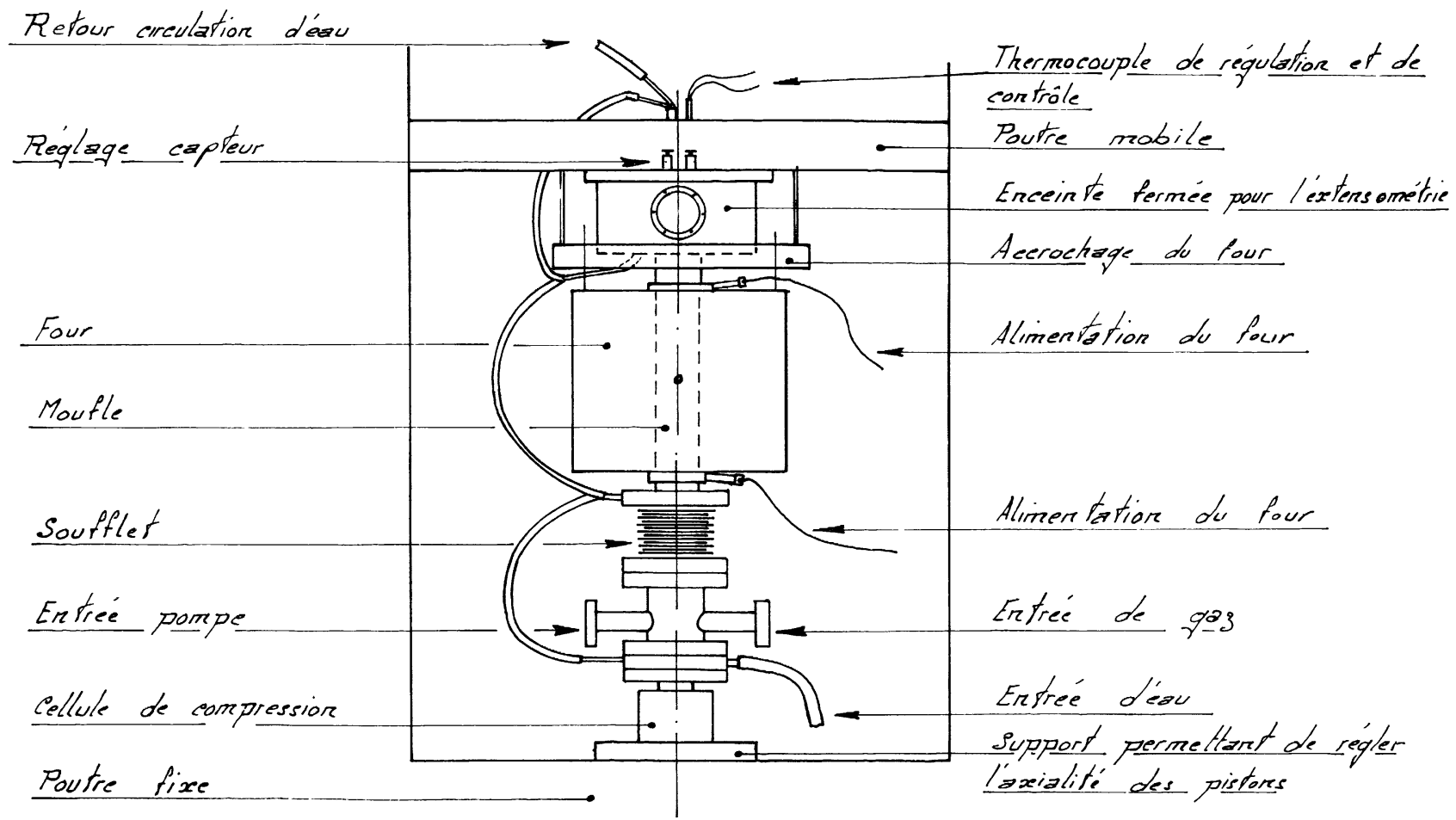

FIG. 1. - Vue schématique du montage.

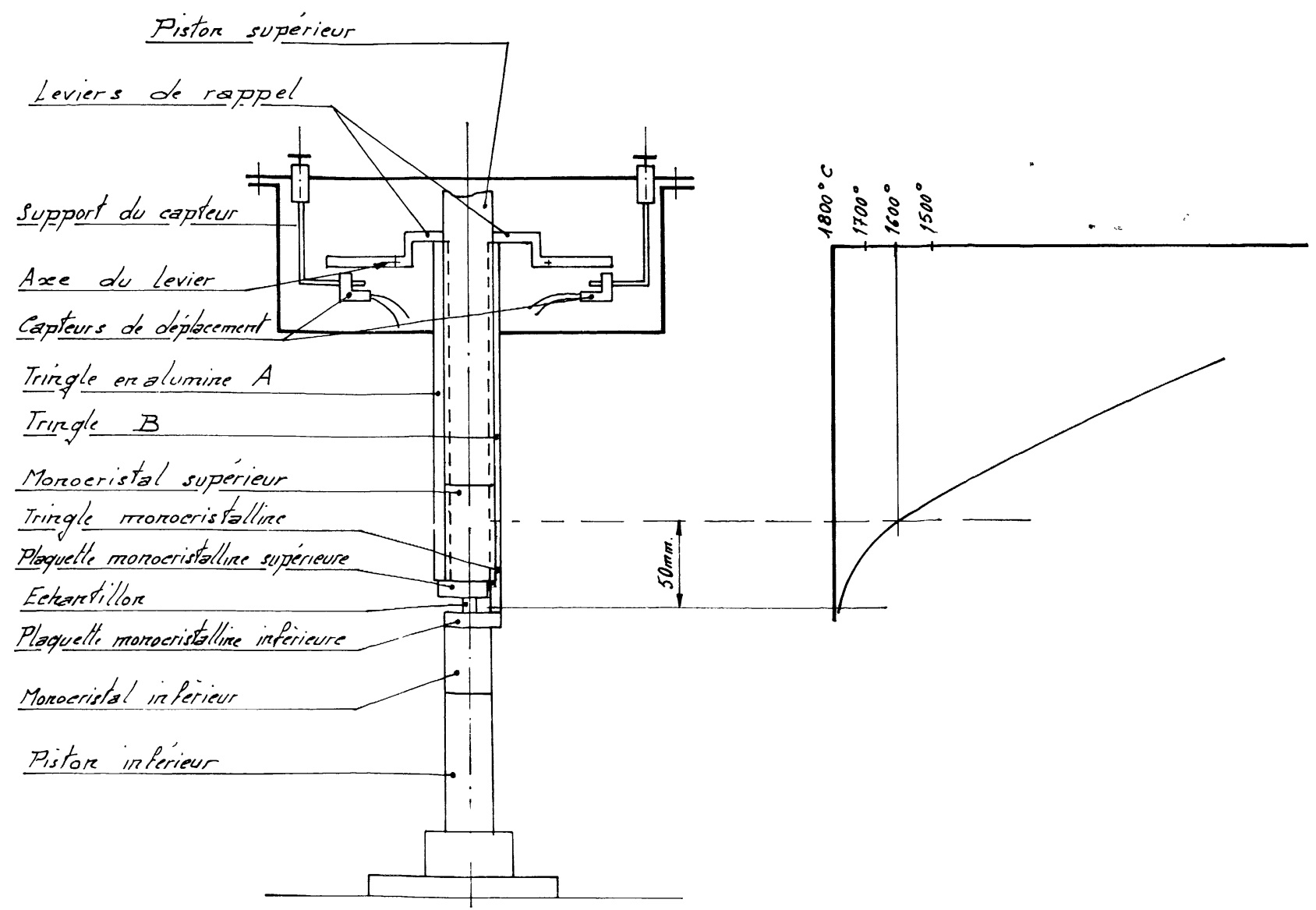

Fig. 2. - Principe de l'extensométrie et profil thermique du four en cours de manipulation à $1800^{\circ} \mathrm{C}$ (la tringle $\mathrm{B}$ est constituée d'éléments monocristallin et polycristallin). 


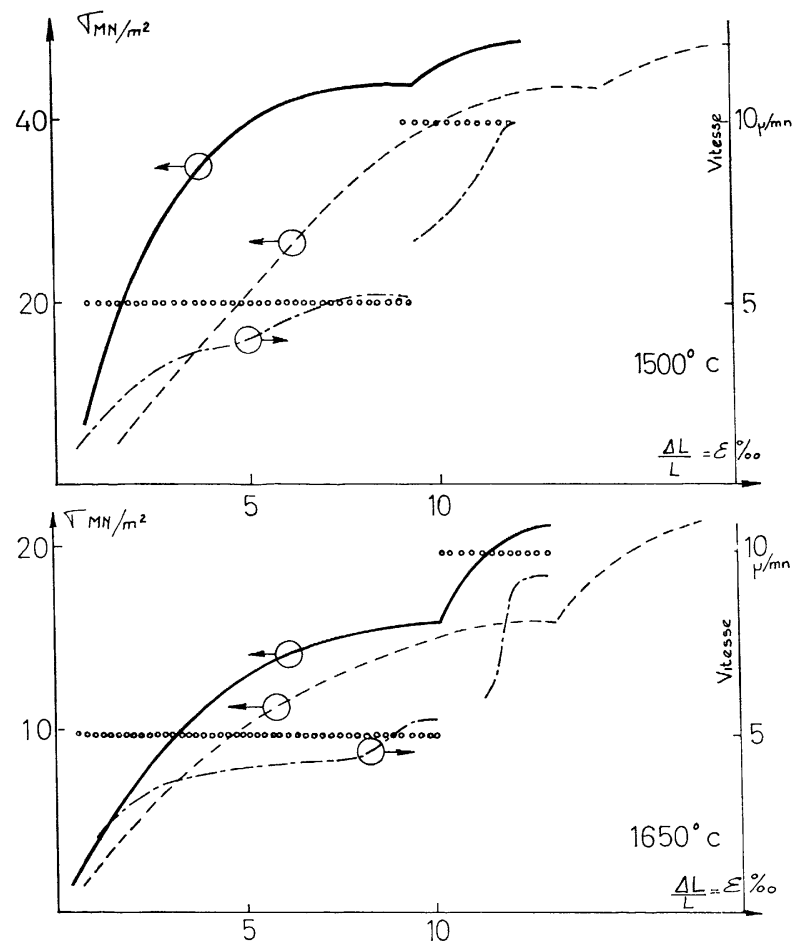

FIG. 3. - Courbe contrainte-déformation nominale à $1500^{\circ} \mathrm{C}$ et $1650^{\circ} \mathrm{C}$ de l'alumine polycristalline utilisée pour fabriquer les pistons, avec changement de la vitesse de traverse $V_{\mathrm{T}}$ de 5 à $10 \mu / \mathrm{min}$. en cours de l'essai :

courbe $\sigma-\varepsilon$ brute calculée en estimant la déformation d'après le déplacement de la traverse.

courbe $\sigma-\varepsilon$ tracée en utilisant les résultats des capteurs de déplacement pour calculer la déformation.

- - - - - vitesse réelle de déplacement des plaquettes monocristallines en fonction de la déformation, calculée à l'aide des capteurs de déplacement.

000000 vitesse de la traverse de la machine INSTRON.

2.2 Extensométrie. - La mesure de la déformation de l'échantillon est faite au niveau des faces de compression afin de ne pas être tributaire des déformations, principalement élastiques, du montage, comme on le serait en estimant cette déformation d'après le déplacement de la poutre mobile ; nous avons placé un système d'extensométrie (Fig. 2): deux tringles en alumine polycristalline prenant appui sur les plaquettes supérieures et inférieures, transmettent leur déplacement par un levier à un capteur de déplacement (marque TESA). La déformation est donnée par la différence des déplacements des tringles $\mathbf{B}$ et $\mathbf{A}$ (Fig. 2). Une sensibilité courante de $0,2 \mu$ est obtenue.

3. Procédure expérimentale. Essais. - 3.1 MoNTAGE. - Les tringles A et B, le monocristal supérieur, la plaquette correspondante ainsi que l'échantillon sont collés au piston supérieur ; ce dernier est fixé à la poutre mobile. Puis, le moufle et le four étant placés, l'ensemble est mis en compression pendant le chauffage afin de maintenir la rigidité du montage sous une charge comprise généralement entre 10 et $20 \mathrm{~N}$ (contrainte sur l'éprouvette de l'ordre de 2 à $4 \mathrm{MN} / \mathrm{m}^{2}$ ).

3.2 RÉsultats eXPérimentauX. - Des essais ont été faits sur des échantillons d'alumine polycristalline, matériau constitutif des pistons, à $1500^{\circ} \mathrm{C}$ et $1650^{\circ} \mathrm{C}$ (Fig. 3).

Le montage d'extensométrie nous a permis de constater que la vitesse de déformation de l'échantillon n'est pas constante au cours de l'essai, et qu'après un saut, celle-ci varie lentement avant d'atteindre sa nouvelle valeur. Ce régime transitoire dépend des paramètres rhéologiques du montage de compression de l'échantillon, par exemple de la facilité du déclenchement du glissement facile [3].

4. Conclusion. - Cet appareillage a été construit pour étudier la déformation à haute température de $\mathrm{Al}_{2} \mathrm{O}_{3}-\alpha$, à une vitesse constante mesurée par extensométrie. Il peut être utilisé pour la déformation d'autres matériaux à condition de résoudre le problème de réactivité chimique avec les pistons en alumine.

Des plans plus détaillés ou tout renseignement relatif à ce montage sont disponibles au laboratoire.

Remerciement. - L'un des auteurs (J. L. C.) remercie la Société Péchiney-Ugine-Kuhlmann pour son soutien financier.

\section{Bibliographie}

[1] Pascoe, R. T., Radford, K. C., Rawlings, R. D., Newey, C. W. A., J. Sci. Instrum. 44 (1967) 366.
[2] Moïse, A., Chim. Actual. 1541-1542 (1974) 78.

[3] CADOz, J. (à paraître). 